\title{
Adesão terapêutica em hipertensos: revisão do papel das novas plataformas através do telemóvel ou smartphone
}

Rodrigo Miguel Loureiro, ${ }^{1}$ Daniela Alves de Azevedo ${ }^{2}$

\section{RESUMO}

Introdução: A pandemia COVID-19 veio comprometer o acompanhamento periódico dos doentes hipertensos em diversos aspetos. Este cenário fez com que se privilegiassem estratégias de telessaúde, como as plataformas $m H$ ealth, que constituem vias alternativas de promoção da autogestão destes doentes. Porém, não existe informação significativa que comprove o seu nível de efetividade. $O$ objetivo deste estudo foi realizar uma revisão sistemática baseada na evidência sobre o papel de plataformas através do telemóvel ou smartphone na adesão de indivíduos com hipertensão ao plano terapêutico proposto.

Métodos: Os artigos desta revisão foram obtidos através da pesquisa bibliográfica em diferentes plataformas científicas, aplicando-se os termos MeSH mobile, phone, hypertension e adherence. Foram incluídos estudos segundo os critérios definidos pelo modelo PICOS: (P) indivíduos adultos com diagnóstico de hipertensão arterial, a realizar pelo menos um fármaco anti-hipertensor; (I) plano terapêutico reforçado com recurso ao telemóvel, telefone fixo ou smartphone; (C) standard care; (O) alterações na adesão terapêutica; $(S)$ revisões sistemáticas e ensaios clínicos aleatorizados controlados. Foi utilizada a escala Strength of Recommendation Taxonomy, da American Academy of Family Physicians, para avaliação dos níveis de evidência e atribuição de forças de recomendação.

Resultados: Dos 729 artigos iniciais foram apenas incluídos sete estudos para a realização desta revisão: uma revisão sistemática e seis ensaios clínicos aleatorizados controlados. Apesar dos estudos relatarem resultados algo heterogéneos e inconsistentes, na generalidade apresentam resultados estatisticamente significativos no sentido do aumento de adesão terapêutica. No entanto, o risco de viés foi relativamente alto.

Conclusões: A evidência atual é de qualidade moderada, algo inconsistente e de difícil generalização, apesar de favorável à efetividade destas plataformas na adesão terapêutica em doentes hipertensos (força de recomendação B). Existe necessidade de maior uniformização e adaptabilidade nos métodos de avaliação da adesão à terapêutica para aumentar a comparabilidade entre estudos e analisar a evolução e tendências numa determinada população.

Palavras-chave: Telefone; Hipertensão arterial; Adesão terapêutica; Telessaúde.

\section{INTRODUÇÃO}

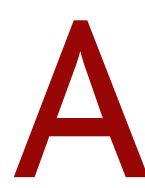
s doenças cardiovasculares (DCV) são uma importante causa de morte e incapacidade a nível mundial, sendo que a hipertensão arterial (HTA) contribui para $45 \%$ do total de mortes por doenças cardíacas e até $51 \%$ das mortes por acidente vascular cerebral. ${ }^{1}$

Em Portugal, as DCV são a principal causa de mortalidade e uma das causas primordiais de morbilidade,

1. USF Novo Norte, Arouca, Portugal.

2. USF Famílias. Lourosa, Portugal. incapacidade e de perda de anos potenciais de vida. $\mathrm{O}$ PROGRAMA NACIONAL PARA AS DOENÇAS CÉREBRO-CARDIOVASCULARES visa atuar a fim de reduzir a mortalidade prematura por DCV como também reduzir a incapacidade que estas causam, nomeadamente através do controlo dos fatores de risco modificáveis, com particular enfoque na HTA. ${ }^{2}$

Estima-se que a HTA tenha sido responsável por 9,4 milhões de mortes e por uma carga de doença de $7 \%$ em 2010. ${ }^{3}$ Em Portugal, em 2014, o Instituto Nacional de Estatística estimou uma prevalência de HTA de $24,5 \%{ }^{4-5}$ 
Os benefícios do controlo da HTA estão documentados e resultam numa importante redução do risco de DCV. O controlo da HTA pode ser conseguido através de um esquema terapêutico farmacológico e/ou não farmacológico eficaz.

O tratamento da HTA com terapêutica anti-hipertensora (TAH) revelou um impacto positivo na saúde dos pacientes, alcançando uma redução de eventos em cerca de 20 a $25 \%$ nas síndromas coronárias agudas, 30 a $35 \%$ em acidentes vasculares cerebrais e $50 \%$ de insuficiência cardíaca. ${ }^{6}$ Embora tenham sido feitos importantes avanços no seu tratamento farmacológico, as taxas de controlo desta doença permanecem baixas e a fraca

\begin{tabular}{|c|c|}
\hline Relacionados com o doente & $\begin{array}{l}\text { Autoeficácia } \\
\text { Esquecimento da toma } \\
\text { Convicções e crenças sobre a doença e/ou medicação } \\
\text { Motivação }\end{array}$ \\
\hline Relacionados com a doença & $\begin{array}{l}\text { Depressão/Stress } \\
\text { Ansiedade } \\
\text { Determinantes físicos } \\
\text { Condição assintomática } \\
\text { Discriminação }\end{array}$ \\
\hline Relacionados com a terapêutica & $\begin{array}{l}\text { Efeitos adversos } \\
\text { Medo de dependência } \\
\text { Complexidade do plano terapêutico }\end{array}$ \\
\hline Relacionados com o sistema de saúde & $\begin{array}{l}\text { Ausência de médico de família } \\
\text { Relação com a equipa de profissionais de saúde } \\
\text { Serviço de saúde }\end{array}$ \\
\hline Socioeconómicos & $\begin{array}{l}\text { Género } \\
\text { Literacia } \\
\text { Faixa etária } \\
\text { Estado civil } \\
\text { Insegurança financeira } \\
\text { Apoio social }\end{array}$ \\
\hline
\end{tabular}
adesão à TAH é considerada como uma das principais causas deste controlo limitado. A adesão medicamentosa é definida na literatura como o grau em que um paciente cumpre a dosagem, a frequência e a posologia dos fármacos prescritos. ${ }^{7-8}$ A não adesão contribui ainda para o aumento dos custos em saúde relacionados com medicamentos, tratamentos e cuidados adequados. ${ }^{6}$

Os métodos usados para aferir a adesão são muito heterogéneos e foram, por isso, já reportados valores de adesão que variam de 20 a $80 \%$ consoante diferentes estudos. ${ }^{6}$ Uma revisão de 2016 apurou que cerca de $45 \%$ dos hipertensos estudados não cumpriam o plano terapêutico. ${ }^{8}$ Estes dados vêm mais uma vez reforçar o quão significativo é o problema da falta de adesão à TAH.

Diferentes estudos revelam uma associação entre este problema e as crenças dos pacientes, características biológicas e socioculturais, representações da doença e do tratamento, relação entre profissional de saúde e utente, suporte familiar, acesso ao sistema de saúde, estatuto socioeconómico, conhecimentos de saúde, raça, etnia, entre outros (Tabela 1$){ }^{6}$

$\mathrm{O}$ acompanhamento rotineiro destes doentes por equipas multidisciplinares, onde o médico de medicina geral e familiar desenvolve um trabalho fundamental, tem um papel importante na promoção da adesão ao tratamento. Diversos estudos têm demonstrado que programas de acompanhamento de hipertensos, maior assiduidade e número de consultas estão associados a uma melhor adesão e a um melhor controlo da PA. É no decorrer deste seguimento que são implementadas as diversas modalidades de intervenção, podendo ser dirigidas aos diferentes tipos de fatores que comprometem a adesão: ao nível do paciente, do profissional de saúde ou do sistema de saúde..$^{9-14}$

A pandemia COVID-19 veio comprometer o acompanhamento periódico de grande parte dos doentes hipertensos em diversos aspetos: desde a desmarcação de consultas de vigilância, redução de disponibilidade de 
contacto com a equipa de saúde, até aos procedimentos burocráticos mais complexos para renovação de medicação. Por outro lado, pela incerteza do futuro, verificou-se que muitos doentes adquiriram a medicação crónica para vários meses, sendo prejudicial na medida em que a TAH pode, a qualquer momento, ser modificada, caso se verifique alteração da sua doença. Por ser uma doença recente e pouco estudada, e dada a facilidade de circulação de informação, surgiu a hipótese de que algumas das TAH pudessem tornar estes doentes mais suscetíveis a infeções graves por este vírus. Apesar deste argumento ter sido posteriormente desmentido pela evidência científica, houve doentes que, com receio de prejuízo para a sua saúde, suspenderam a terapêutica. ${ }^{15-18}$

Apesar do panorama, a adaptação dos médicos de família e respetivos utentes a esta nova realidade foi quase imediata, tendo sido privilegiadas e reforçadas as estratégias de telessaúde. Esta pode definir-se como a prestação de cuidados de saúde à distância através do uso de telecomunicações, sendo que pode variar de intervenções simples, como um contacto telefónico entre o paciente e o profissional de saúde, ou algo mais elaborado como o uso de novas tecnologias que promovem a autogestão. ${ }^{19-20}$

A OMS define autogestão como a capacidade dos indivíduos de gerir os seus próprios problemas de saúde, com ou sem o apoio de profissionais ou recursos de saúde. ${ }^{21}$ Os métodos de autogestão incluem a educação para a saúde, monitorização dos próprios dados clínicos e comportamentais (e.g., dieta, exercício, tabagismo e consumo de álcool), titulação do contacto com recursos de saúde e suporte na adesão ao plano terapêutico. $^{22}$

Surgiu uma nova plataforma que já mostrou benefícios na promoção da autogestão em diversas doenças crónicas: a mHealth. A OMS definiu, pela primeira vez, o termo mHealth em 2010 como a promoção da autogestão através de dispositivos eletrónicos móveis, como telemóveis, smartphones, dispositivos de monitorização de pacientes e dispositivos sem fio. ${ }^{23}$ Desde então, a tecnologia mHealth tem-se tornado cada vez mais amplamente disponível e sofisticada. ${ }^{24-25}$

A mHealth, por empregar uma variedade de diferentes recursos, incluindo mensagens de texto convencional (SMS - Short Message System), e-mails, telefonemas e aplicações para telemóvel, possibilita a superação de barreiras geográficas, aumentando, assim, o acesso e prestação de serviços de saúde a populações e comunidades carentes. Porém, não existe informação significativa que comprove o seu nível de efetividade. ${ }^{26-28}$

O objetivo deste estudo foi realizar uma revisão sistemática baseada na evidência sobre o papel das plataformas através do telemóvel ou smartphone na adesão de indivíduos com HTA ao plano terapêutico proposto.

\section{MÉTODOS}

Esta revisão sistemática foi estruturada de acordo com o modelo Preferred reporting items for systematic reviews and meta-analyses (PRISMA) e a atribuição dos níveis de evidência (NE) e forças de recomendação segundo a escala Strength of Recommendation Taxonomy (SORT). ${ }^{29-30}$

A pesquisa bibliográfica foi realizada durante os meses de maio e junho de 2020, através das seguintes bases de dados ou plataformas eletrónicas: National Guideline Clearinghouse, National Institute for Health and Care Excellence Guidelines Finder, Direção-Geral da Saúde, The Database of Abstracts of Reviews of Effects (DARE), The Cochrane Library, Turning Research Into Practice Database e MEDLINE. A pesquisa incidiu sobre artigos publicados nos últimos cinco anos. Adicionalmente foram analisadas as referências bibliográficas de alguns artigos, de forma a identificar bibliografia relevante. Durante a pesquisa aplicaram-se os termos MeSH mobile, phone, hypertension e adherence e foram incluídos os estudos publicados nos idiomas inglês ou português. Após este processo foram obtidos um total de 729 artigos.

Após a eliminação de artigos em duplicado procedeu-se à seleção dos artigos cujo título ou resumo apresentavam conteúdo relevante para a revisão em causa.

Relativamente aos critérios de elegibilidade utilizou-se o modelo PICOS (Population, Intervention, Comparison, Outcome, Studies) para listar e definir todas as variáveis procuradas nos vários estudos analisados: (1) a população-alvo deste estudo foi a dos indivíduos adultos com diagnóstico de HTA, a realizar pelo menos um fármaco anti-hipertensor; (2) a intervenção correspondeu a um plano terapêutico reforçado com recurso ao telemóvel, telefone fixo ou smartphone; (3) a 
comparação foi realizada com o plano terapêutico implementado de modo padrão em ambiente de consulta presencial (usual ou standard care); (4) o outcome avaliado incidiu sobre alterações na adesão ao plano terapêutico proposto; (5) o tipo de estudo incluído para este trabalho foram as revisões sistemáticas (RS) e os ensaios clínicos aleatorizados controlados (ECAC). Não foi definido um período mínimo de follow-up para a inclusão dos estudos.

Excluíram-se os artigos cujo propósito não fosse a avaliação da adesão ao plano terapêutico e aqueles em que apenas o resumo estava disponível para consulta.

A avaliação da qualidade metodológica dos artigos foi debatida entre os autores e apresentada de forma descritiva, tendo em conta as características da amostra, da intervenção e do comparador, o método de aleatorização e ocultação, o tempo de seguimento e a medição da adesão e dos resultados. Os processos de pesquisa, seleção e recolha dos dados importantes dos estudos incluídos foram realizados exclusivamente pelos autores.

\section{RESULTADOS}

\section{Características gerais dos estudos}

Dos 729 artigos iniciais foram incluídos apenas sete estudos para a realização desta revisão: uma $\mathrm{RS}^{31} \mathrm{e}$ seis ECAC $^{32-37}$ escritos em inglês e realizados nos Estados Unidos da América (EUA), Chile, Gana, África do Sul e China entre 2015 e 2020. O processo de seleção dos estudos apresenta-se no fluxograma da Figura 1 e o resumo das respetivas características, bem como o nível de evidência atribuído segundo a escala SORT, apresentam-se nas Tabelas 2 e 3, respetivamente.

A população amostral total foi de 10.321 pessoas, variando entre 18 e 8.933 indivíduos entre estudos. Verificou-se que em todos os estudos havia predomínio de

mulheres. A idade dos pacientes nos sete artigos variou de 36 a 78 anos. A duração conjunta da intervenção e follow-up variou de seis semanas a 18 meses. Metade dos projetos durou menos de seis meses.

Todos os estudos recorreram a intervenções através de plataformas telefónicas como ferramentas para aumentar a adesão terapêutica em doentes hipertensos.

A intervenção no grupo de controlo adotada foi a de usual ou standard care, não sendo especificada em grande parte dos estudos.

Em termos de outcome, seis dos estudos avaliaram simultaneamente alterações nos valores de PA sistólica (PAS) e diastólica (PAD), controlo tensional alvo e adesão terapêutica. Apenas Varleta e colaboradores (2017) ${ }^{34}$ avaliaram exclusivamente a adesão terapêutica.

\section{Características das intervenções}

Verificaram-se diversos tipos de intervenções através de diferentes plataformas móveis - SMS, aplicações de smartphones, dispositivos externos ligados ao telemóvel - que permitem monitorização. Através destes meios, os investigadores proporcionaram educação para a saúde, fornecendo desde informação sobre a HTA, a importância e estratégias de mudança de estilo 


\section{TABELA 2. Resumo das características dos ensaios clínicos aleatorizados controlados (ECAC) incluídos nesta revisão ${ }^{32-37}$}

\begin{tabular}{|c|c|c|c|c|}
\hline $\begin{array}{l}\text { Autores } \\
\text { (país, data) }\end{array}$ & $\begin{array}{l}\text { Idade e género } \\
\text { da população }\end{array}$ & Grupo da intervenção (GI) & Grupo de controlo (SC) & Outcome \\
\hline $\begin{array}{l}\text { Davidson } \\
\text { et al. (EUA, } \\
\text { 2015) }\end{array}$ & $\begin{array}{l}36 \text { a } 60 \text { anos } \\
15 \text { indivíduos } \\
\text { do sexo } \\
\text { masculino (M) } \\
\text { e } 23 \text { indivíduos } \\
\text { do sexo } \\
\text { feminino (F) }\end{array}$ & $\begin{array}{l}n=18 \\
\text { Intervenção: aplicação de telemóvel (SMASH, } \\
\text { Smartphone Medication Adherence Stops } \\
\text { Hypertension) que notifica e motiva os } \\
\text { pacientes a fazer medicação e a fazer } \\
\text { controlo de valores de PA. } \\
\text { Notificação consoante a compliance. } \\
\text { Duração da intervenção: } 6 \text { meses }\end{array}$ & $\begin{array}{l}n=20 \\
\text { Intervenção: Standard } \\
\text { Care (não especificado) } \\
\text { Duração da intervenção: } \\
6 \text { meses }\end{array}$ & $\begin{array}{l}\text { Alterações nos valores } \\
\text { de PA sistólica e } \\
\text { diastólica, controlo } \\
\text { tensional alvo e } \\
\text { adesão terapêutica }\end{array}$ \\
\hline $\begin{array}{l}\text { Kim et al. } \\
\text { (EUA, } \\
2016)\end{array}$ & $\begin{array}{l}49 \text { a } 66 \text { anos } \\
27 \mathrm{M} \mathrm{e} 68 \mathrm{~F}\end{array}$ & $\begin{array}{l}n=52 \\
\text { Intervenção: aplicação de telemóvel } \\
\text { (HealthyCircles App) que motiva e } \\
\text { recomenda os pacientes a adotar estilos } \\
\text { de vida saudáveis, a aderir à medicação e } \\
\text { a controlar os valores de PA regularmente. } \\
\text { Notificação consoante a compliance. } \\
\text { Duração da intervenção: } 6 \text { meses }\end{array}$ & $\begin{array}{l}n=43 \\
\text { Intervenção: Standard } \\
\text { Care (não claro, embora } \\
\text { refiram que os } \\
\text { participantes tinham } \\
\text { acesso a informação } \\
\text { sobre a doença, medidas } \\
\text { de prevenção e sugestões } \\
\text { de estratégias a } \\
\text { implementar) } \\
\text { Duração da intervenção: } \\
6 \text { meses }\end{array}$ & $\begin{array}{l}\text { Alterações nas } \\
\text { escolhas } \\
\text { comportamentais } \\
\text { (número de cigarros } \\
\text { por dia, frequência } \\
\text { de consumo de } \\
\text { bebidas alcoólicas, } \\
\text { períodos de exercício } \\
\text { físico), nos valores de } \\
\text { PA sistólica e diastólica, } \\
\text { controlo tensional alvo } \\
\text { e adesão terapêutica }\end{array}$ \\
\hline $\begin{array}{l}\text { Varleta et } \\
\text { al. (Chile, } \\
\text { 2017) }\end{array}$ & $\begin{array}{l}49 \text { a } 71 \text { anos } \\
51 \mathrm{M} \text { e } 240 \mathrm{~F}\end{array}$ & $\begin{array}{l}n=151 \\
\text { Intervenção: envio de SMS com conteúdo } \\
\text { educacional sobre alimentação, consumo } \\
\text { salino e adesão terapêutica a cada } 12 \text { dias. } \\
\text { Duração da intervenção: } 6 \text { meses }\end{array}$ & $\begin{array}{l}n=140 \\
\text { Intervenção: Standard } \\
\text { Care (não especificado) } \\
\text { Duração da intervenção: } \\
6 \text { meses }\end{array}$ & $\begin{array}{l}\text { Alterações na adesão } \\
\text { terapêutica e } \\
\text { alterações nos valores } \\
\text { de PA sistólica e } \\
\text { diastólica. }\end{array}$ \\
\hline $\begin{array}{l}\text { Sarfo et } \\
\text { al. } \\
\text { (Gana, } \\
\text { 2018) }\end{array}$ & $\begin{array}{l}42 \text { a } 68 \text { anos } \\
51 \mathrm{M} \text { e } 240 \mathrm{~F}\end{array}$ & $\begin{array}{l}n=28 \\
\text { Intervenção: envio de SMS com conteúdo } \\
\text { educacional sobre alterações do estilo de vida } \\
\text { (não especificados) e conteúdo motivacional } \\
\text { para aumento de adesão. } \\
\text { Periodicidade não especificada. } \\
\text { Duração da intervenção: } 9 \text { meses }\end{array}$ & $\begin{array}{l}n=27 \\
\text { Intervenção: Usual Care } \\
\text { e envio de SMS com } \\
\text { conteúdo educacional } \\
\text { sobre alterações do } \\
\text { estilo de vida (não } \\
\text { especificados) } \\
\text { Periodicidade não } \\
\text { especificada } \\
\text { Duração da intervenção: } \\
9 \text { meses }\end{array}$ & $\begin{array}{l}\text { Alterações nos } \\
\text { valores de PA } \\
\text { sistólica e } \\
\text { diastólica, controlo } \\
\text { tensional alvo e } \\
\text { adesão terapêutica }\end{array}$ \\
\hline
\end{tabular}

de vida (alimentação, consumos nocivos, exercício físico) até à motivação e alerta sobre os benefícios de uma boa compliance terapêutica. Por outro lado, estas plataformas permitiam um registo dos valores de PA, fornecendo em tempo real feedback positivo e lembretes sobre o cumprimento adequado do plano 


\begin{tabular}{|c|c|c|c|c|}
\hline $\begin{array}{l}\text { Autores } \\
\text { (país, data) }\end{array}$ & $\begin{array}{l}\text { Idade e género } \\
\text { da população }\end{array}$ & Grupo da intervenção (GI) & Grupo de controlo (SC) & Outcome \\
\hline $\begin{array}{l}\text { Bobrow } \\
\text { et al. } \\
\text { (África do } \\
\text { Sul, 2016) }\end{array}$ & $\begin{array}{l}43 \text { a } 71 \text { anos } \\
379 \text { M e } 993 \mathrm{~F}\end{array}$ & $\begin{array}{l}n=914(457+457) \\
\text { Intervenção: } \\
\text { Braço A: Usual Care + envio de SMS interativa } \\
\text { com informação sobre HTA e estilo de vida } \\
\text { saudável com periodicidade semanal, com } \\
\text { possibilidade de esclarecer dúvidas; } \\
\text { Braço B: Usual Care + envio de SMS com } \\
\text { informação sobre HTA, estilos de vida } \\
\text { saudáveis e adesão terapêutica com } \\
\text { periodicidade semanal. } \\
\text { Duração da intervenção: } 12 \text { meses }\end{array}$ & $\begin{array}{l}n=458 \\
\text { Intervenção: Usual Care } \\
\text { (informação escrita } \\
\text { sobre HTA e tratamento) } \\
\text { Periodicidade não } \\
\text { especificada } \\
\text { Duração da intervenção: } \\
12 \text { meses }\end{array}$ & $\begin{array}{l}\text { Alterações nos valores } \\
\text { de PA média, controlo } \\
\text { tensional alvo e } \\
\text { adesão terapêutica }\end{array}$ \\
\hline
\end{tabular}

\section{Risco de viés e qualidade}

As Tabelas 4 e 5 apresentam a avaliação crítica dos estudos incluídos nesta revisão e a atribuição dos respetivos NE. Verificou-se que, no geral, o risco de viés foi relativamente alto, dado que nenhum estudo é absolutamente livre de viés.

Sessenta e seis por cento dos ECAC incluídos revelaram ausência ou não especificação dos métodos de ocultação, nomeadamente dos participantes, profissionais ou dos avaliadores de outcome (viés de performance). Verificou-se uma ocultação da aleatorização, follow-up adequado (variando entre 91,6 e 100\%) e recurso a avaliação estatística adequada e clara em todos os artigos incluídos. ${ }^{32-37}$

$\mathrm{Na}$ revisão de Li e colaboradores, ${ }^{31}$ oito artigos incluídos foram classificados como baixo risco para viés de seleção; no entanto, os restantes apresentaram metodologia pouco clara ou não especificada, como os métodos de randomização e alocação, ocultação dos avaliadores ou a descrição do que constituía a intervenção realizada no grupo de controlo. Em oito dos es- tudos verificaram baixo risco de atrito (ou de perda de seguimento); no entanto, também registaram que quatro dos estudos incluídos constataram taxas de abandono superiores a $15 \%$. Em termos de viés de relato (ou de resultados), os autores verificaram um baixo risco em 22 dos 24 artigos incluídos.

\section{CONCLUSÕES}

A problemática da adesão à TAH tem vindo a apresentar uma importância crescente ao influenciar o controlo da doença, a morbilidade e mortalidade associadas, a qualidade de vida dos pacientes e os custos em saúde. Esta revisão sistemática foi realizada com a finalidade de avaliar o impacto das novas plataformas na adesão terapêutica em doentes com HTA.

Os resultados, apesar de algo heterogéneos e inconsistentes, na generalidade apresentam resultados positivos e, por vezes, significativos, no sentido do aumento da adesão terapêutica.

A heterogeneidade de resultados pode ser explicada pela variabilidade de diversos parâmetros dos estudos: 
No grupo de intervenção verificou-se redução significativa dos valores de PAS $(-8,99 \mathrm{mmHg}$ no $\mathrm{Gl}$ e apenas $-5,92 \mathrm{mmHg}$ no SC; $p<0,05)$ e PAD $(-7,04 \mathrm{mmHg}$ no $\mathrm{Gl}$ e apenas $-4,14 \mathrm{mmHg} ;<<0,05)$, alcance e manutenção dos valores de PA dentro dos valores alvo (aumento de $38 \%$ no Gl e de $28 \%$ no SC; $p=0,011$ ) e aumento da adesão terapêutica $(p=0,004)$

(1) características da amostra variam em termos de dimensão, género, naturalidade da população, situação social, económica e cultural do país na qual a amostra se insere; (2) apesar de todos os estudos cumprirem o critério de recorrerem a plataformas telefónicas e fomentarem o aumento de adesão terapêutica, as características de operacionalidade variaram consideravelmente entre elas; (3) a duração do estudo foi outro fator de flutuação; (4) a disparidade entre metodologias, recursos e materiais não permite uma adequada comparação e reprodução. A estratificação destas intervenções ajudará a produzir estimativas e conclusões mais precisas e úteis.

Noutro prisma, o forte poder estatístico, o follow-up adequado e as características consensuais de intervenção no grupo de controlo entre grande parte dos estudos são os pontos fortes e positivos dos estudos considerados nesta revisão. É de salientar que nenhum dos estudos relatou redução da adesão com este tipo de novas plataformas.

Ainda no ponto de vista metodológico, a maioria dos estudos recorreu a meios de self-report: escalas e questionários dirigidos aos doentes para a recolha dos dados sobre adesão à terapêutica. Tendo em conta que o fracasso da terapêutica se deve substancialmente à inadequada adesão ao tratamento, é importante a utilização de um instrumento válido e fiável de self-report. A utilização de escalas e questionários pode ser justificada pela sua simplicidade de aplicação e reduzidos custos. No entanto, trata-se de formas suscetíveis de viés de compliance porque podem ser altamente influenciados e facilmente distorcidos pelos participantes dada a sua subjetividade. São ainda métodos que não permitem obtenção fácil de informação quando a amostra é elevada, limitando a extrapolação dos resultados obtidos.

A versão mais recente da Morisky Medication Adherence Scale (MMAS) foi o método mais frequentemente utilizado na avaliação da adesão terapêutica nos estudos incluídos. Neste questionário de self-report, originalmente desenvolvido em 1986 num estudo que incluiu apenas doentes hipertensos e composto apenas por quatro itens de resposta dicotómica («Sim» ou "Não») para determinação da adesão à terapêutica, concluiu-se que apresentava uma sensibilidade de $43,6 \%$ e uma especificidade de $81,4 \%$. O MMAS-8 é a versão atualizada e aperfeiçoada deste questionário, desenvolvido de forma a minimizar diversos vieses. ${ }^{38-39}$ Moon e colaboradores, em 2017, ${ }^{40}$ realizaram uma revisão sistemática com meta-análise da versão mais atual deste questionário e verificaram que, com a adição das novas questões, a sensibilidade e a especificidade alcançaram um valor sumário de 74\% (95\% IC $68 \% \sim 79 \%$ ) e $43 \%$ (95\% IC 32\% 53\%) respetivamente. No entanto, na análise dos estudos que incluíram a sua revisão verificou-se que a sensibilidade variou entre $13 \%$ (95\% IC 6\% 24\%) e 94\% (95\% IC 74\% 99\%) e a especificidade variou entre 55\% (95\% IC 46\% 65\%) e $86 \%$ (95\% IC 76\% 93\%); e, mais especificamente no subgrupo dos doentes hipertensos, a sensibilidade foi de $43 \%$ (95\% IC 26 61\%) e a especificidade de $71 \%$ (95\% IC 62\% 79\%). Os autores concluíram que há necessidade de demonstração, adequação e validação para uma determinada população-alvo antes que se possa aplicar o MMAS-8. Por outro lado, o MMAS-8 não permite avaliar o uso incorreto da terapêutica, dado que não aborda o tempo ou a via de administração. ${ }^{40}$

Existe, portanto, uma necessidade de maior uniformização e adaptabilidade nos métodos de avaliação da adesão à terapêutica para aumentar a comparabilida- 


\section{TABELA 3. Resumo das características da revisão sistemática incluída nesta revisão ${ }^{31}$}

\begin{tabular}{|c|c|c|c|c|c|}
\hline $\begin{array}{l}\text { Autores } \\
\text { (país, } \\
\text { data) }\end{array}$ & $\begin{array}{l}\text { Estudos } \\
\text { incluídos }\end{array}$ & $\begin{array}{c}\text { Caracterização da } \\
\text { população }\end{array}$ & Metodologia & Conclusões & Limitações \\
\hline $\begin{array}{l}\text { Li et al. } \\
\text { (China, } \\
\text { 2020) }\end{array}$ & $\begin{array}{l}\text { Revisão } \\
\text { baseada na } \\
\text { evidência, } \\
\text { composta } \\
\text { por } 24 \\
\text { ECAC e } \\
\text { uma meta- } \\
\text {-análise com } \\
12 \text { ECAC }\end{array}$ & $\begin{array}{l}\text { População escolhida: } \\
\text { indivíduos adultos com } \\
\text { diagnóstico de HTA } \\
\text { ( } n=8933) \\
\text { Amostras variaram } \\
\text { entre os } 54 \text { e os } 1.372 \\
\text { indivíduos } \\
\text { Idade da população: } \\
\text { entre os } 44 \text { e os } 78 \\
\text { anos }\end{array}$ & $\begin{array}{l}\text { Avaliação da influência } \\
\text { de plataformas mHealth } \\
\text { (mensagens de texto, } \\
\text { caixas de medicamentos } \\
\text { inteligentes, plataformas } \\
\text { online e chamadas de } \\
\text { voz interativas) nos } \\
\text { valores de PA sistólica } \\
\text { e diastólica, controlo } \\
\text { tensional alvo e adesão } \\
\text { terapêutica } \\
\text { Inclusão de ECAC entre } \\
2010 \text { e } 2019 \\
\text { Duração da intervenção } \\
\text { variou de } 1,5 \text { a } 18 \\
\text { meses }\end{array}$ & $\begin{array}{l}\text { De acordo com a meta- } \\
\text {-análise verificou-se } \\
\text { uma redução mais } \\
\text { significativa na PAS } \\
(p<0,001) \text { e PAD } \\
(p<0,001) \text { no grupo de } \\
\text { intervenção com } \\
\text { ferramentas de } \\
\text { telemedicina quando } \\
\text { comparados com o } \\
\text { grupo de controlo } \\
\text { Um total de } 16 \text { estudos } \\
\text { concluiu que houve } \\
\text { melhoria na adesão ao } \\
\text { plano terapêutico no } \\
\text { grupo de intervenção }\end{array}$ & $\begin{array}{l}\text { Tamanho da amostra, } \\
\text { metodologia, tempo } \\
\text { de follow-up muito } \\
\text { heterogéneos entre os } \\
\text { estudos incluídos } \\
\text { Metodologia não clara } \\
\text { ou reportada em cerca } \\
\text { de metade dos artigos } \\
\text { incluídos } \\
13 \text { dos estudos } \\
\text { incluídos não } \\
\text { especificaram o tempo } \\
\text { de follow-up } \\
\text { Apenas } 12 \text { estudos } \\
\text { avaliavam a adesão } \\
\text { terapêutica } \\
\text { exclusivamente e } \\
\text { apenas sete revelaram } \\
\text { significância estatística }\end{array}$ \\
\hline
\end{tabular}

\section{TABELA 4. Avaliação do risco de viés para os ECAC ${ }^{32-37}$}

\begin{tabular}{|c|c|c|c|c|c|c|c|}
\hline ECAC & $\begin{array}{c}\text { Ocultação } \\
\text { aleatorização }\end{array}$ & $\begin{array}{c}\text { Ocultação } \\
\text { participantes }\end{array}$ & $\begin{array}{c}\text { Ocultação } \\
\text { profissionais }\end{array}$ & $\begin{array}{l}\text { Ocultação } \\
\text { avaliadores } \\
\text { de outcome }\end{array}$ & Follow-up & $\begin{array}{c}\text { Poder } \\
\text { estatístico } \\
\text { adequado }\end{array}$ & $\begin{array}{l}\text { Nível de } \\
\text { evidência }\end{array}$ \\
\hline $\begin{array}{l}\text { Davidson et al. } \\
\text { (EUA, 2015) }\end{array}$ & Sim & $\begin{array}{c}\text { Não } \\
\text { reportado }\end{array}$ & $\begin{array}{c}\text { Não } \\
\text { reportado }\end{array}$ & $\begin{array}{c}\text { Não } \\
\text { reportado }\end{array}$ & $\operatorname{Sim}(100 \%)$ & Sim & 2 \\
\hline $\begin{array}{l}\text { Kim et al. } \\
\text { (EUA, 2016) }\end{array}$ & Sim & Não & Não & Não & $\operatorname{Sim}(100 \%)$ & Sim & 2 \\
\hline $\begin{array}{l}\text { Varleta et al. } \\
\text { (Chile, 2017) }\end{array}$ & Sim & Sim & Sim & Sim & $\operatorname{Sim}(92,6 \%)$ & Sim & 2 \\
\hline $\begin{array}{l}\text { Sarfo et al. } \\
\text { (Gana, 2018) }\end{array}$ & Sim & Não & Não & Não & $\operatorname{Sim}(91,6 \%)$ & Sim & 2 \\
\hline $\begin{array}{l}\text { Bobrow et al. } \\
\text { (África do Sul, } \\
2016 \text { ) }\end{array}$ & Sim & Sim & Sim & Sim & $\operatorname{Sim}(92 \%)$ & Sim & 2 \\
\hline $\begin{array}{l}\text { Gong et al. } \\
\text { (China, 2020) }\end{array}$ & Sim & $\begin{array}{c}\text { Não } \\
\text { reportado }\end{array}$ & $\begin{array}{c}\text { Não } \\
\text { reportado }\end{array}$ & $\begin{array}{c}\text { Não } \\
\text { reportado }\end{array}$ & $\operatorname{Sim}(92,3 \%)$ & Sim & 2 \\
\hline
\end{tabular}




TABELA 5. Avaliação crítica da revisão sistemática ${ }^{31}$
\begin{tabular}{|l|c|c|c|c|c|c|}
\hline RBE & $\begin{array}{c}\text { Definição } \\
\text { de PICO }\end{array}$ & $\begin{array}{c}\text { Métodos de } \\
\text { pesquisa } \\
\text { apropriados }\end{array}$ & $\begin{array}{c}\text { Inclusão de } \\
\text { estudos } \\
\text { relevantes }\end{array}$ & $\begin{array}{c}\text { Avaliação } \\
\text { crítica dos } \\
\text { estudos }\end{array}$ & $\begin{array}{c}\text { Ausência de } \\
\text { heterogeneidade }\end{array}$ & $\begin{array}{c}\text { Nível de } \\
\text { evidência }\end{array}$ \\
\hline $\begin{array}{l}\text { Li et al. } \\
\text { (China, 2020) }\end{array}$ & $\operatorname{Sim}$ & $\operatorname{Sim}$ & $\operatorname{Sim}$ & $\operatorname{Sim}$ & Não & 1 \\
\hline
\end{tabular}

de entre estudos e analisar a evolução e tendências numa determinada população.

Com esta revisão conclui-se que a evidência atual é de qualidade moderada, algo inconsistente e de difícil generalização, apesar de favorável à efetividade destas plataformas na adesão terapêutica em doentes hipertensos (força de recomendação B).

A evidência que suporta estas recomendações resume-se essencialmente a seis ECAC e uma RS, cuja qualidade é heterogénea e a generalização dos resultados e respetiva aplicabilidade é limitada. Esta fraqueza foi também fomentada pela restrição da pesquisa de artigos em inglês ou português e pela disponibilidade gratuita do texto integral dos artigos, reduzindo, assim, a diversidade dos estudos a analisar.

Após a realização desta revisão extensível a diversas plataformas de artigos científicos, os autores acreditam que esta é a primeira revisão sistemática nacional que avaliou esta temática. Os resultados positivos de efetividade na maioria destes ensaios devem ser motivo de reflexão, de forma a fomentar futuras investigações acerca da utilidade destas plataformas inovadoras como ferramentas adicionais de adesão terapêutica. É de salientar que estas plataformas são meras ferramentas adjuvantes ao trabalho desenvolvido pelo médico de família, sendo apenas um complemento a adotar no futuro no seguimento de doentes hipertensos.

No futuro, será necessário realizar novos estudos com estruturas e metodologia mais específicas, claras e consensuais, de forma a alcançar conclusões de qualidade: (1) ao nível da intervenção e outcome é pertinente analisar simultaneamente o custo-efetividade do método de intervenção, de forma a avaliar a exequibilidade da sua adoção generalizada; (2) ainda no campo do outcome, a adesão terapêutica deve ser qualificada/quantificada através de um método consensual de avaliação que, ao mesmo tempo, seja válido para a população-alvo e permita margem mínima para possíveis vieses.

\section{REFERÊNCIAS BIBLIOGRÁFICAS}

1. Lim SS, Vos T, Flaxman AD, Danaei G, Shibuya K, Adair-Rohani H, et al. A comparative risk assessment of burden of disease and injury attributable to 67 risk factors and risk factor clusters in 21 regions, 19902010: a systematic analysis for the Global Burden of Disease Study 2010. Lancet. 2012;380(9859):2224-60.

2. Direção-Geral da Saúde. Programa nacional para as doenças cérebrocardiovasculares. Lisboa: DGS; 2017.

3. World Health Organization. Global status report on noncommunicable diseases 2014: attaining the nine global noncommunicable diseases targets - a shared responsibility [homepage]. Geneva:WHO; 2015. Available from: https://reliefweb.int/report/world/global-status-reportnoncommunicable-diseases-2014-attaining-nine-global

4. Instituto Nacional de Estatística. Inquérito nacional de saúde 2014 [homepage]. Lisboa: INE; 2016. Available from: https://www.ine.pt/xurl/ pub/263714091

5. Polonia J, Martins L, Pinto F, Nazare J. Prevalence, awareness, treatment and control of hypertension and salt intake in Portugal: changes over a decade - The PHYSA study. J Hypertens. 2014;32(6):1211-21.

6. Gosmanova EO, Kovesdy CP. Adherence to antihypertensive medications: is prescribing the right pill enough? Nephrol Dial Transplant. 2015;30(10):1649-56.

7. Direção-Geral da Saúde. Abordagem terapêutica da hipertensão arterial: norma n. ${ }^{\circ}$ 026/2011, de 29/09/2011, atualizado em 19/03/2013. Lisboa: DGS; 2013.

8. Abegaz TM, Shehab A, Gebreyohannes EA, Bhagavathula AS, Elnour AA. Nonadherence to antihypertensive drugs: a systematic review and meta-analysis. Medicine. 2017;96(4):e5641.

9. Khatib R, Schwalm JD, Yusuf S, Haynes RB, McKee M, Khan M, et al. Patient and healthcare provider barriers to hypertension awareness, treatment and follow up: a systematic review and meta-analysis of qualitative and quantitative studies. PLoS One. 2014;9(1):e84238.

10. Jankowska-Pola ska B, Uchmanowicz I, Dudek K, Mazur G. Relationship between patients' knowledge and medication adherence among patients with hypertension. Patient Prefer Adherence. 2016;10:2437-47.

11. Vrijens B, Antoniou S, Burnier M, de la Sierra A, Volpe M. Current situation of medication adherence in hypertension. Front Pharmacol. 2017;8:100. 
12. Morrison VL, Holmes EA, Parveen S, Plumpton CO, Clyne W, De Geest $S$, et al. Predictors of self-reported adherence to antihypertensive medicines: a multinational, cross-sectional survey. Value Health. 2015;18(2):206-16.

13. AlGhurair SA, Hughes CA, Simpson SH, Guirguis LM. A systematic review of patient self-reported barriers of adherence to antihypertensive medications using the World Health Organization Multidimensional Adherence Model. J Clin Hypertens. 2012;14(12):877-86.

14. Maimaris W, Paty J, Perel P, Legido-Quigley H, Balabanova D, Nieuwlaat $R$, et al. The influence of health systems on hypertension awareness, treatment, and control: a systematic literature review. PLoS Med. 2013;10(7):e1001490.

15. Gurwitz D. Angiotensin receptor blockers as tentative SARS CoV 2 therapeutics. Drug Dev Res. 2020;81(5):534-40.

16. Gheblawi M, Wang K, Viveiros A, Nguyen Q, Zhong JC, Turner AJ, et al. Angiotensin-converting enzyme 2: SARS-CoV-2 receptor and regulator of the renin-angiotensin system: celebrating the 20th anniversary of the discovery of ACE2. Circ Res. 2020;126(10):1456-74.

17. Zheng YY, Ma YT, Zhang JY, Xie X. COVID-19 and the cardiovascular system. Nat Rev Cardiol. 2020;17(5):259-60.

18. South AM, Tomlinson L, Edmonston D, Hiremath S, Sparks MA. Controversies of renin-angiotensin system inhibition during the COVID19 pandemic. Nat Rev Nephrol. 2020;16(6):305-7.

19. Peacock E, Krousel-Wood M. Adherence to antihypertensive therapy. Med Clin North Am. 2017;101(1):229-45.

20. Sivakumaran D, Earle KA. Telemonitoring: use in the management of hypertension. Vasc Health Risk Manag. 2014;10:217-24.

21. World Health Organization. WHO consolidated guideline on self-care interventions for health: sexual and reproductive health and rights [homepage]. Geneva:WHO; 2019. Available from: https://www.ncbi.nlm. nih.gov/books/NBK544164/

22. Bodenheimer T, Lorig K, Holman H, Grumbach K. Patient self-management of chronic disease in primary care. JAMA. 2002;288(19):2469-75.

23. World Health Organization. Global diffusion of eHealth: making universal health coverage achievable [homepage]. Geneva: WHO; 2016. Available from: https://apps.who.int/iris/handle/10665/252529

24. World Health Organization. Atlas eHealth country profiles: based on the findings of the second global survey on eHealth [homepage]. Geneva: WHO; 2011. Available from: https://apps.who.int/iris/handle/10665/44502

25. World Health Organization. mHealth: new horizons for health through mobile technologies [homepage]. Geneva:WHO; 2011. Available from: https://apps.who.int/iris/handle/10665/44607

26. Cruz-Zapata B, Fernández-Alemán JL, Idri A, Toval A. Empirical studies on usability of mHealth apps: a systematic literature review. J Med Syst. 2015;39(2):1.

27. Morawski K, Ghazinouri R, Krumme A, McDonough J, Durfee E, Oley L, et al. Rationale and design of the Medication adherence Improvement Support App For Engagement—Blood Pressure (MedISAFE-BP) trial.Am Heart J. 2017; 186:40-7.

28. Williams B, Mancia G, Spiering W, Rosei EA, Azizi M, Burnier M, et al. 2018 ESC/ESH Guidelines for the management of arterial hypertension. Eur Heart J. 2018;39(33):3021-104.

29. Liberati A, Altman DG, Tetzlaff J, Mulrow C, Gøtzsche PC, loannidis JP, et al. The PRISMA statement for reporting systematic reviews and meta-analyses of studies that evaluate health care interventions: explanation and elaboration. PLoS Med. 2009;6(7):e1000100.

30. Ebell MH, Siwek J, Weiss BD, Woolf SH, Susman J, Ewigman B, et al. Strength of recommendation taxonomy (SORT): a patient-centered approach to grading evidence in the medical literature. J Am Board Fam Pract. 2004;17(1):59-67.

31. Li R, Liang N, Bu F, Hesketh T. The effectiveness of self-management of hypertension in adults using mobile health: systematic review and meta-analysis. JMIR Mhealth Uhealth. 2020;8(3):e17776.

32. Davidson TM, McGillicuddy J, Mueller M, Brunner-Jackson B, Favella A, Anderson $A$, et al. Evaluation of an mHealth medication regimen selfmanagement program for African American and Hispanic uncontrolled hypertensives. J Pers Med. 2015;5(4):389-405.

33. Kim JY, Wineinger NE, Steinhubl SR. The influence of wireless self-monitoring program on the relationship between patient activation and health behaviors, medication adherence, and blood pressure levels in hypertensive patients: a substudy of a randomized controlled trial. J Med Internet Res. 2016;18(6):e116.

34. Varleta P, Acevedo M,Akel C, Salinas C, Navarrete C, García A, et al. Mobile phone text messaging improves antihypertensive drug adherence in the community. J Clin Hypertens. 2017;19(12):1276-84.

35. Sarfo FS, Treiber F, Gebregziabher M, Adamu S, Nichols M, Singh A, et al. Phone-based intervention for blood pressure control among Ghanaian stroke survivors: a pilot randomized controlled trial. Int J Stroke. 2019;14(6):630-8.

36. Bobrow K, Farmer AJ, Springer D, Shanyinde M, Yu LM, Brennan T, et al. Mobile phone text messages to support treatment adherence in adults with high blood pressure (SMS-text Adherence Support [StAR]): a single-nlind, randomized trial. Circulation. 2016;133(6):592-600.

37. Gong K, Yan YL, Li Y, Du J, Wang J, Han Y, et al. Mobile health applications for the management of primary hypertension: a multicenter, randomized, controlled trial. Medicine. 2020;99(16):e19715.

38. Morisky DE, Ang A, Krousel-Wood M, Ward HJ. Predictive validity of a medication adherence measure in an outpatient setting. J Clin Hypertens. 2008;10(5):348-54.

39. Voils $\mathrm{Cl}$, Hoyle RH, Thorpe CT, Maciejewski ML, Yancy Jr WS. Improving the measurement of self-reported medication nonadherence.J Clin Epidemiol. 2011;64(3):250-4.

40. Moon SJ, Lee WY, Hwang JS, Hong YP, Morisky DE. Accuracy of a screening tool for medication adherence: a systematic review and meta-analysis of the Morisky Medication Adherence Scale-8. PLoS One. 2017;12(11):e0187139.

\section{CONFLITO DE INTERESSES}

Os autores declaram não possuir quaisquer conflitos de interesse.

\section{ENDEREÇO PARA CORRESPONDÊNCIA}

Rodrigo Miguel Loureiro

E-mail: rodrigo.mrsp.loureiro@gmail.com

https://orcid.org/0000-0001-7619-4604

Recebido em 01-08-2020

Aceite para publicação em 08-02-2021 


\section{ABSTRACT \\ THERAPEUTIC ADHERENCE OF HYPERTENSIVE PATIENTS: A REVIEW OF THE NEW MOBILE PHONE OR SMARTPHONE PLATFORMS' ROLE}

Introduction: COVID-19 pandemic compromised hypertensive patients monitoring in several aspects. This scenario emphasized telehealth strategies like phone consultation. mHealth is another emerging way to promote patient self-management through mobile devices. However, there is no significant information to prove its effectiveness. The purpose of this paper was to carry out an evidence-based systematic review on mobile phone or smartphone platforms' role on therapeutic adherence of individuals with hypertension.

Methods: The articles were obtained through bibliographic research on different scientific platforms using the MeSH terms 'mobile', 'phone', 'hypertension' and 'adherence'. Studies were included according to the following PICOS model criteria: (P) adult individuals diagnosed with arterial hypertension, taking at least one antihypertensive drug; $(\mathrm{I})$ reinforced therapeutic plan using a cell phone, landline or smartphone platform; (C) standard care; $(\mathrm{O})$ changes in therapeutic adherence; (S) systematic reviews and randomized controlled clinical trials. The American Academy of Family Physicians Strength of Recommendation Taxonomy scale was used to assess levels of evidence and to assign the recommendation force.

Results: Of the 729 initial articles, only seven studies were included in this review: one systematic review and six randomized controlled clinical trials. Although most studies reported somewhat heterogeneous and inconsistent results, they presented statistically significant results in terms of increasing therapeutic adherence. However, the risk of bias was relatively high.

Conclusions: Current evidence is of moderate, inconsistent, and difficult to generalize quality, despite being favourable to the effectiveness of these platforms increasing therapeutic adherence in hypertensive patients (strength of recommendation B). Greater methodologic uniformity and adaptability are needed when assessing therapy adherence in order to increase comparability between studies and to analyse its evolution in a given population.

Keywords: Hypertension; Adherence; Phone; Smartphone. 